\title{
Abelian groups gradings on null-filiform and one-parametric filiform Leibniz algebras
}

\author{
Antonio Jesús Calderón ${ }^{a}$, Luisa María Camacho ${ }^{b}$, Ivan Kaygorodov ${ }^{c, d} \&$ Bakhrom Omirov $^{e}$ \\ ${ }^{a}$ Dpto. Matemáticas. Universidad de Cádiz. 11510 Puerto Real, Cádiz, Spain \\ ${ }^{b}$ Dpto. Matemática Aplicada I. Universidad de Sevilla. Avda. Reina Mercedes, s/n. 41012 Sevilla, Spain \\ ${ }^{c}$ CMCC, Universidade Federal do ABC, Santo André - SP, Brazil \\ ${ }^{d}$ Moscow Center for Fundamental and Applied Mathematics, Moscow, GSP-1, 119991, Russia \\ ${ }^{e}$ National University of Uzbekistan, Institute of Mathematics Uzbekistan Academy of Sciences, Tashkent 100174, Uzbekistan \\ E-mail addresses: \\ Antonio Jesús Calderón (ajesus.calderon@uca.es) \\ Luisa María Camacho (lcamacho@us.es) \\ Ivan Kaygorodov (kaygorodov.ivan@gmail.com) \\ Bakhrom Omirov (omirovb@mail.ru)
}

\begin{abstract}
We classify, up to equivalences, all abelian groups gradings on null-filiform and oneparametric filiform Leibniz algebras. Any grading on a null-filiform Leibniz algebra is toral but there are non-toral gradings on one-parametric filiform Leibniz algebras.
\end{abstract}

MSC2020: 17A32, 17B30, 17B40.

Key words: Leibniz algebra, nilpotent algebra, grading, automorphism, torus.

\section{INTRODUCTION}

Gradings by abelian groups have played a key role in the study of Lie algebras and superalgebras, starting with the root space decomposition of the semisimple Lie algebras over the complex field, which is an essential ingredient in the Killing-Cartan classification of these algebras. Gradings by a cyclic group appear in the connection between Jordan algebras and Lie algebras through the TitsKantor-Koecher construction, and in the theory of Kac-Moody Lie algebras. Gradings by the integers or the integers modulo 2 are ubiquitous in Geometry. Also there are some papers about non-group gradings [7, 30].

In 1989, Patera and Zassenhaus [29] began a systematic study of gradings by abelian groups on Lie algebras. They raised the problem of classifying the fine gradings, up to equivalence, on the simple Lie algebras over the complex numbers. This problem has been settled now thanks to the work of many colleagues. After that, gradings of simple alternative and simple Malcev algebras [17], the simple Kac Jordan superalgebra [5], countless simple Lie algebras [6, 11, -16, 18,-22] and filiform Lie algebras [4] were described.

The concept of length of a Lie algebra was introduced by Gómez, Jiménez-Merchán and Reyes in [23, 24]. They distinguished an interesting family: algebras admitting a grading with the greatest possible number of non-zero subspaces. Actually, the gradings with a large number of non-zero subspaces enable us to describe the multiplication on the algebra more exactly.

In the past years, Leibniz algebras have been under active research (see, for example, [1, 3, 8,-10, 25-27]). The main result on the structure of finite-dimensional Leibniz algebras asserts that a Leibniz algebra decomposes into a semidirect sum of a solvable radical and a semisimple Lie algebra [3]. Therefore, the main problem of the description of finite-dimensional Leibniz algebras consists of the

\footnotetext{
${ }^{1}$ The work was supported by the PCI of the UCA 'Teoría de Lie y Teoría de Espacios de Banach', the PAI with project numbers FQM298, FQM7156, Ministerio de Economía y Competitividad (Spain), grant MTM2016-79661-P (AEI/FEDER, UE, support included) and MTM2013-41208P, the 2014-2020 ERDF Operational Programme and by the Department of Economy, Knowledge, Business and University of the Regional Government of Andalusia FEDER-UCA18107643, RFBR 20-01-00030, CNPq 302980/2019-9.
} 
study of solvable Leibniz algebras. Similarly to the case of Lie algebras the study of solvable Leibniz algebras is reduced to nilpotent ones [9].

Since the description of all $n$-dimensional nilpotent Leibniz algebras is an unsolvable task (even in the case of Lie algebras), we have to study nilpotent Leibniz algebras under certain conditions (conditions on index of nilpotency, various types of grading, characteristic sequence etc.) [1, 26, 27]. The well-known natural grading of nilpotent Lie and Leibniz algebras is very helpful when investigating of the properties of those algebras without restrictions on the grading. Indeed, we can always choose a homogeneous basis and thus the grading allows to obtain more explicit conditions for the structural constants. Moreover, such grading is useful for the investigation of cohomologies for the considered algebras, because it induces the corresponding grading of the group of cohomologies. Thus, it is very crucial to know what kind of grading admits a nilpotent Leibniz algebra.

In the present paper we begin the study of gradings on Leibniz algebras by classifying, up to equivalence, of all abelian groups gradings of null-filiform and one-parametric filiform Leibniz algebras.

\section{PRELiminARIES}

In this section we give necessary definitions and preliminary results.

Definition 1. A vector space with a bilinear multiplication $(L, \cdot)$ over the complex field is called a Leibniz algebra if for any $x, y, z \in L$ the so-called Leibniz identity

$$
x(y z)=(x y) z-(x z) y
$$

holds.

For a given Leibniz algebra $L$ the sequence of two-sided ideals defined recursively as follows:

$$
L^{1}=L, L^{k+1}=L^{k} L, k \geq 1,
$$

is said to be the lower central series of $L$.

Definition 2. A Leibniz algebra $L$ is said to be nilpotent, if there exists $n \in \mathbb{N}$ such that $L^{n}=0$. The minimal number $n$ with such property is said to be the index of nilpotency of the algebra $L$.

Definition 3. An n-dimensional Leibniz algebra L is said to be null-filiform if $\operatorname{dim} L^{i}=n+1-i, 1 \leq$ $i \leq n+1$.

Theorem 4 ( [2]). An arbitrary complex n-dimensional null-filiform non-Lie Leibniz algebra is isomorphic to the algebra

$$
N F_{n}: \quad e_{i} e_{1}=e_{i+1}, \quad 1 \leq i \leq n-1
$$

where $\left\{e_{1}, e_{2}, \ldots, e_{n}\right\}$ is a basis of the algebra $N F_{n}$.

Actually, a nilpotent Leibniz algebra is null-filiform if and only if it is one-generated algebra. Notice that this notion has no sense in Lie algebra case, because they are at least two-generated.

Definition 5. An n-dimensional Leibniz algebra $L$ is said to be filiform if $\operatorname{dim} L^{i}=n-i$, for $2 \leq i \leq$ $n$.

Definition 6. Let $G$ be a group. An algebra $L$ is a $G$-graded algebra if and only if the vector space $L$ has the following decomposition $L=\bigoplus_{g \in G} L_{g}$ and the multiplication law of $L$ has the following property $L_{g} L_{h} \subset L_{g+h}, \forall g, h \in G$. Any $L_{g}$ is called a homogeneous subspace and the set of all $g$ such that $L_{g} \neq 0$ is called the support of the grading. 
For a $G$-graded algebra $L=\bigoplus_{g \in G} L_{g}$ we will use the following notation $L_{g}:=\left\langle e_{i_{1}}, \ldots, e_{i_{k}}\right\rangle_{g}$, if the homogeneous subspace $L_{g}$ is generated by $e_{i_{1}}, \ldots, e_{i_{k}}$.

Definition 7. Given two groups gradings $L=\bigoplus_{g \in G} L_{g}$ and $L=\bigoplus_{h \in H} L_{h}$ we shall say that they are equivalent if there are:

(1) a bijection $\sigma: G \rightarrow G^{\prime}$ between the supports of the first and second gradings respectively,

(2) an algebra automorphism $\varphi$ of $L$ such that $\varphi\left(L_{g}\right)=L_{\sigma(g)}$ for any $g \in G$.

Following [28] we introduce the next class of filiform non-Lie Leibniz algebras.

Definition 8. A complex $n$-dimensional algebra admitting a basis $\left\{e_{1}, e_{2}, \ldots, e_{n}\right\}$ such that the table of multiplication of the algebra has the following form:

$$
\begin{aligned}
& e_{i} e_{1}=e_{i+1}, \quad 2 \leq i \leq n-1, \\
& e_{1} e_{2}=\theta e_{n},
\end{aligned}
$$

with $\theta \in \mathbb{C}$ is called a one-parametric filiform Leibniz algebra.

In the description of all abelian groups grading of our algebras, we are using the techniques explained in the recent monograph [21, Section 1.4], which have been successfully used in the classification of abelian groups gradings on different classes of algebras (see for instance [4, 5, 13, 14]). To do that, we will always suppose that the grading group is generated by the support of the grading. Roughly speaking, it is shown in the above references that any group grading, (with the group finitely generated abelian), is induced by a finitely generated abelian subgroup of diagonalizable automorphisms of the automorphism group of the algebra, (finite dimensional and over an algebraically closed field of characteristic zero), under study. The homogeneous components are the simultaneous eigenspaces relative to the given subgroup of automorphisms. Furthermore, up to equivalences of gradings, any such subgroup is contained in the normalizer of some fixed maximal torus of the automorphism group of the algebra.

A special kind of gradings arises when we consider the inducing automorphisms not only in the normalizer of a maximal torus, but in the torus itself.

Definition 9. A grading of an algebra is said to be toral if it is produced by automorphisms within a torus of the automorphism group of the algebra.

\section{GRADINGS ON NULL-FILIFORM LEIBNIZ ALGEBRAS}

By the above, in the first we are calculating the group of automorphisms of our algebra. In the second, we are proving that the normalizer of a maximal torus in the group of automorphisms is the same torus and so all of our gradings will be toral ones. Finally, we are constructing all toral gradings on our algebra.

Let $A:=N F_{n}$ be a null-filiform Leibniz algebra.

3.1. Automorphisms of $A$. Let $f \in \operatorname{Aut}(A)$, then $f\left(e_{1}\right)=a e_{1}+\sum_{j=2}^{n} a_{j} e_{j}$, and

$$
f\left(e_{i}\right)=f\left(e_{i-1} e_{1}\right)=\ldots=f\left(\left(\ldots\left(\left(e_{1} e_{1}\right) e_{1}\right) \ldots\right) e_{1}\right)=\left(\ldots\left(\left(f\left(e_{1}\right) f\left(e_{1}\right)\right) f\left(e_{1}\right)\right) \ldots\right) f\left(e_{1}\right),
$$

hence $f\left(e_{i}\right)=a^{i} e_{i}+a^{i-1} \sum_{j=1}^{n-i} a_{j+1} e_{j+i}, \quad 1 \leq i \leq n$, with $a \neq 0$. It is easy to see that all automorphisms of $A$ have the same form. 
3.2. Maximal Torus. It is easy to verify that a maximal torus is formed by:

$$
\mathcal{T}=\left\{t_{a}:=\left(\begin{array}{cccc}
a & 0 & \ldots & 0 \\
0 & a^{2} & \ldots & 0 \\
\vdots & \vdots & \ddots & \vdots \\
0 & 0 & \ldots & a^{n}
\end{array}\right): a \in \mathbb{K}^{*}\right\} \cong \mathbb{K}^{*}
$$

Lemma 10. Let $\mathcal{N}(\mathcal{T})$ be the normalizer of $\mathcal{T}$. Then, $\mathcal{N}(\mathcal{T})=\mathcal{T}$.

Proof. We have $\mathcal{N}(\mathcal{T})=\left\{M \in \operatorname{Aut}(A): M T M^{-1} \in \mathcal{T}, \forall T \in \mathcal{T}\right\}$.

For

$$
M=\left(\begin{array}{lllll}
a & 0 & \ldots & 0 & 0 \\
a_{2} & a^{2} & \ldots & 0 & 0 \\
\vdots & \vdots & \ddots & \vdots & \vdots \\
a_{n-1} & a a_{n-2} & \ldots & a^{n-1} & 0 \\
a_{n} & a a_{n-1} & \ldots & a^{n-1} a_{2} & a^{n}
\end{array}\right) \text { and } T=\left(\begin{array}{lllll}
\lambda & 0 & \ldots & 0 & 0 \\
0 & \lambda^{2} & \ldots & 0 & 0 \\
\vdots & \vdots & \ddots & \vdots & \vdots \\
0 & 0 & \ldots & \lambda^{n-1} & 0 \\
0 & 0 & \ldots & 0 & \lambda^{n}
\end{array}\right)
$$

we have

$$
M T=\left(\begin{array}{lllll}
a \lambda & 0 & \ldots & 0 & 0 \\
a_{2} \lambda & a^{2} \lambda^{2} & \ldots & 0 & 0 \\
\vdots & \vdots & \ddots & \vdots & \vdots \\
a_{n-1} \lambda & a a_{n-2} \lambda^{2} & \ldots & a^{n-1} \lambda^{n-1} & 0 \\
a_{n} \lambda & a a_{n-1} \lambda^{2} & \ldots & a^{n-1} a_{2} \lambda^{n-1} & a^{n} \lambda^{n}
\end{array}\right)
$$

We need to prove the existence of $T^{\prime} \in \mathcal{T}$ such that $M T=T^{\prime} M$. For

$$
T^{\prime}=\left(\begin{array}{llll}
d & 0 & \ldots & 0 \\
0 & d^{2} & \ldots & 0 \\
\vdots & \vdots & \ddots & \vdots \\
0 & 0 & \ldots & d^{n}
\end{array}\right)
$$

we conclude that $d=\lambda$ and by choosing $\lambda \neq 1,0$ we get $a_{2}=a_{3}=\cdots=a_{n}=0$ and $M \in \mathcal{T}$.

3.3. Cyclic gradings. A cyclic grading is a toral grading produced by a single toral element $t_{a}$. In this case the grading is always equivalent to a grading by a cyclic group. In order to study the grading induced by $t_{a}$ on $A$, which is the decomposition of $A$ as a direct sum of eigenspaces of such toral element $t_{a}$, we are going to distinguish different possibilities motivated by the cardinal of the possible values of the set of eigenvalues of $t_{a}$.

We distinguish the following cases:

- $a^{i} \neq 1$ for $i=1,2, \ldots, n$. In this case, we have the next grading:

$$
A=\left\langle e_{1}\right\rangle_{1} \oplus\left\langle e_{2}\right\rangle_{2} \oplus \cdots \oplus\left\langle e_{n}\right\rangle_{n}: \mathbb{Z}_{\text {-grading }}
$$

- $a^{i}=1$ where $a$ is an $i$-primitive root of 1 , and $1 \leq i \leq n-1, i \in \mathbb{N}$. If $i=1$, we obtain the trivial grading. Thus, we can consider $2 \leq i \leq n-1$. Let us write $n=m i+p$ with $0 \leq p \leq i-1, p \in \mathbb{N}$. We have a $\mathbb{Z}_{i}$-grading with the following homogeneous subspaces: 


$$
\begin{aligned}
A_{\overline{0}} & =\left\langle e_{i}, e_{2 i}, \ldots, e_{m i}\right\rangle \\
A_{\overline{1}} & =\left\langle e_{1}, e_{i+1}, \ldots, e_{m i+1}\right\rangle \\
A_{\overline{2}} & =\left\langle e_{2}, e_{i+2}, \ldots, e_{m i+2}\right\rangle \\
\ldots & \ldots \\
A_{\bar{p}}= & \left\langle e_{p}, e_{i+p}, \ldots, e_{m i+p}\right\rangle \\
A_{\overline{p+1}}=\left\langle e_{p+1}, e_{i+p+1}, \ldots, e_{(m-1) i+p+1}\right\rangle & \ldots \\
A_{\overline{i-1}} & =\left\langle e_{i-1}, e_{2 i-1}, \ldots, e_{(m-1) i+i-1}\right\rangle .
\end{aligned}
$$

Lemma 11. Let $A$ be a null-filiform Leibniz algebra of dimension $n$. Then any cyclic grading is equivalent to only one of the following $n$ gradings:

(I) The trivial grading given by $A=\left\langle e_{1}, e_{2}, \ldots, e_{n}\right\rangle$.

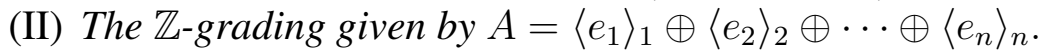

(III) For any $2 \leq i \leq n-1$, the $\mathbb{Z}_{i^{-}}$grading given by $A=A_{\overline{0}} \oplus A_{\overline{1}} \oplus \cdots \oplus A_{\overline{i-1}}$, where homogeneous subspaces are described in grading (1), being $n=m i+p$ with $0 \leq p \leq i-1$.

Proof. We have only to show that two different gradings in the lemma are not equivalent, but this is an immediate consequence of the fact that the cardinal of the support of any grading is different to the cardinal of the support of any other different grading.

\subsection{Classification theorem.}

Lemma 12. If $e_{1}$ is a homogeneous element of an abelian group grading (that is, $e_{1} \in A_{x}$ for some $x \in G)$, then the grading is one of the list of Lemma 11

Proof. Let $e_{1} \in A_{x}$ and $x \in G$ be. Let $i$ be the order of $x$.

- $i>n$. Let $j \leq n$ be, we have $e_{j}=\left(\ldots\left((e_{1} \underbrace{\left.\left.\left.e_{1}\right) e_{1}\right) \ldots\right) e_{1}}_{j-1} \in A_{j x}\right.\right.$ and the $\mathbb{Z}$-grading

$$
A=\left\langle e_{1}\right\rangle \oplus\left\langle e_{2}\right\rangle \oplus \cdots \oplus\left\langle e_{n}\right\rangle .
$$

- $i \leq n$. Then, $e_{1} \in A_{x}, e_{2} \in A_{2 x}, \ldots, e_{i-1} \in A_{(i-1) x}$ with $|\{x, 2 x, \ldots,(i-1) x\}|=i-1$. Let $j \geq i$ be, $j=i m+p$ with $0 \leq p<i$. Then $e_{j}=\left(\ldots\left((e_{1} \underbrace{\left.\left.\left.e_{1}\right) e_{1}\right) \ldots\right) e_{1}}_{j-1} \in A_{i m+p}=A_{\bar{p}}\right.\right.$. Thus,

$$
A=\left\langle e_{1}, e_{1+i}, \ldots\right\rangle_{\overline{1}} \oplus\left\langle e_{2}, e_{2+i}, \ldots\right\rangle_{\overline{2}} \oplus \cdots \oplus\left\langle e_{i-1}, e_{2 i-1}, \ldots\right\rangle_{\overline{i-1}} \oplus\left\langle e_{i}, e_{2 i}, \ldots\right\rangle_{\overline{0}}
$$

Lemma 10 and Lemma 12 will allow us to assert that all of the abelian group gradings of $A$ are the ones given in Lemma 11. Indeed, since by Lemma 10, $\mathcal{N}(\mathcal{T})=\mathcal{T}$, we know that all of the gradings are toral. Hence any grading is the simultaneous eigenspaces decomposition of $A$ respect to a given (abelian) subgroup of semisimple automorphisms $\mathcal{U}$ contained in $\mathcal{T}$. Now. since

$$
\mathcal{T}=\left\{t_{a}: a \in \mathbb{K}^{*}\right\}
$$

(see 3.1.2), and any $t_{a}$ is of the form $t_{a}:=\left(\begin{array}{cccc}a & 0 & \ldots & 0 \\ 0 & a^{2} & \ldots & 0 \\ \vdots & \vdots & \ddots & \vdots \\ 0 & 0 & \ldots & a^{n}\end{array}\right)$ respect to the basis $\left\{e_{1}, e_{2}, \ldots, e_{n}\right\}$ we have that $e_{1}$ belongs to the eigenspace associated to the eigenvalue $a$, for any $t_{a} \in \mathcal{T}$ and so $e_{1}$ belongs to some simultaneous eigenspace respect to the decomposition of $A$ relative to the simultaneous 
eigenspaces decomposition of $A$ through the abelian family of semisimple automorphisms $\mathcal{U}$. From here $e_{1}$ is a homogeneous element of any abelian group grading of $A$ and we can apply Lemma 12 to conclude the next theorem:

Theorem 13. Any abelian group grading of a null-filiform Leibniz algebra is equivalent to only one of the list of Lemma 11

\section{GRADINGS ON ONE-PARAMETRIC FILIFORM LEIBNIZ ALGEBRAS}

We will follow the same program than in the previous section to classify the abelian group gradings on one-parametric filiform Leibniz algebras. First we compute the group of automorphisms of our algebras. Then, in order to find a maximal torus and compute its normalizer, we will have two distinguish two cases attending to the possible nullity of the parameter. We will obtain that just in case this parameter is zero all of the gradings are toral ones. In this case we will also have that any abelian group grading is necessarily cyclic. For the remaining cases we will have to develop new tools for their study.

4.1. Automorphisms of $F_{1}$. Our first goal is to compute its group of automorphisms. $\operatorname{Let} \operatorname{dim}\left(F_{1}\right) \geq$ 4 and $f \in \operatorname{Aut}\left(F_{1}\right)$. Then

$$
f\left(e_{1}\right)=\sum_{k=1}^{n} a_{k} e_{k} \text { and } f\left(e_{2}\right)=\sum_{k=1}^{n} b_{k} e_{k} .
$$

It is easy to see that $a_{1} b_{2} \neq 0$. From $f\left(e_{1}\right)^{2}=f\left(e_{1}^{2}\right)=0$ we find $f\left(e_{1}\right)=a_{1} e_{1}+a_{n} e_{n}$; and from $f\left(e_{2}\right)^{2}=f\left(e_{2}^{2}\right)=0$ we have $b_{1}=0$. Also from $f\left(e_{1}\right) f\left(e_{2}\right)=\theta f\left(e_{n}\right)$ we obtain $\theta\left(1-a_{1}^{n-3}\right)=0$.

If we rename $a_{1}=a$ and $b_{2}=b$, we have

$$
\begin{aligned}
f\left(e_{1}\right) & =a e_{1}+a_{n} e_{n} \\
f\left(e_{i}\right) & =a^{i-2}\left(b e_{i}+b_{3} e_{i+1}+\cdots+b_{n-i+2} e_{n}\right), 2 \leq i \leq n, \quad a b \neq 0, \\
\theta\left(1-a^{n-3}\right) & =0 .
\end{aligned}
$$

So we get:

$$
\operatorname{Aut}\left(F_{1}\right)=\left\{\left(\begin{array}{lllll}
a & 0 & 0 & \ldots & 0 \\
0 & b & 0 & \ldots & 0 \\
0 & b_{3} & a b & \ldots & 0 \\
0 & b_{4} & a b_{3} & \ldots & 0 \\
\vdots & \vdots & \vdots & \ddots & \vdots \\
a_{n} & b_{n} & a b_{n-1} & \ldots & a^{n-2} b
\end{array}\right): \begin{array}{c}
a_{n}, b_{3}, \ldots, b_{n} \in \mathbb{C} \text { and } a, b \in \mathbb{C}^{*}, \quad \theta\left(1-a^{n-3}\right)=0 \\
\text {. }
\end{array}\right\} .
$$

To calculate a maximal torus in the above group of automorphisms we are going to distinguish two cases.

4.2. Case in which $\theta=0$. Since in this case

$$
\operatorname{Aut}\left(F_{1}\right)=\left\{\left(\begin{array}{lllll}
a & 0 & 0 & \ldots & 0 \\
0 & b & 0 & \ldots & 0 \\
0 & b_{3} & a b & \ldots & 0 \\
0 & b_{4} & a b_{3} & \ldots & 0 \\
\vdots & \vdots & \vdots & \ddots & \vdots \\
a_{n} & b_{n} & a b_{n-1} & \ldots & a^{n-2} b
\end{array}\right): a_{n}, b_{3}, \ldots, b_{n} \in \mathbb{C}, a, b \in \mathbb{C}^{*}\right\}
$$


for $n \geq 3$ (the case $n=3$ is easy to verify), we get that a maximal torus is formed by:

$$
\mathcal{T}=\left\{t_{a, b}:=\left(\begin{array}{ccccc}
a & 0 & 0 & \ldots & 0 \\
0 & b & 0 & \ldots & 0 \\
0 & 0 & a b & \ldots & 0 \\
\vdots & \vdots & \vdots & \ddots & \vdots \\
0 & 0 & 0 & \ldots & a^{n-2} b
\end{array}\right): a, b \in \mathbb{C}^{*}\right\} \cong \mathbb{C}^{*} \times \mathbb{C}^{*}
$$

By similar calculations to Lemma 10 one can prove the following result:

Lemma 14. Let $\mathcal{N}(\mathcal{T})$ be the normalizer of $\mathcal{T}$. Then, $\mathcal{N}(\mathcal{T})=\mathcal{T}$.

Denote by $A$ a one-parametric filiform Leibniz algebra with $\theta=0$. Let us compute the grading on $A$ induced by only an element $t_{a, b}$ in our maximal torus. We will denote $d_{1}=a, d_{2}=b$ and $d_{i}=a^{i-2} b$ with $3 \leq i \leq n$ the diagonal of the matrix $t_{a, b}$ respect our fixed basis.

We distinguish the following cases:

(1) $a=b$.

(1.1) If $a=b=1$. In this case, we have the trivial grading:

$$
A=\left\langle e_{1}, e_{2}, \ldots, e_{n}\right\rangle \text {. }
$$

(1.2) If $a=b$ and $a^{i}=1$, being $a$ an $i$-primitive root of 1 and $2 \leq i \leq n-1, i \in \mathbb{N}$. Let $n=i m+p$ be with $0 \leq p<i$. Then, we get the $\mathbb{Z}_{i}$-grading

$$
\begin{aligned}
& A=\quad\left\langle e_{i+1}, \quad e_{2 i+1} \quad \ldots, e_{1+(m-1) i}, \quad e_{1+m i}\right\rangle_{\overline{0}}, \\
& \oplus\left\langle e_{1}, e_{2}, \quad e_{2+i}, \quad e_{2+2 i}, \quad \ldots, \quad e_{2+(m-1) i}, \quad e_{2+m i}\right\rangle_{\overline{1}} \\
& \oplus\left\langle e_{3}, \quad e_{3+i}, \quad e_{3+2 i}, \quad \ldots, \quad e_{3+(m-1) i}, \quad e_{3+m i}\right\rangle \overline{2} \\
& \oplus\left\langle e_{p}, \quad e_{p+i}, \quad e_{p+2 i}, \quad \ldots, \quad e_{p+(m-1) i}, \quad e_{p+m i}\right\rangle_{\overline{p-1}} \\
& \oplus\left\langle e_{p+1}, \quad e_{p+1+i}, \quad e_{p+1+2 i}, \quad \ldots, \quad e_{p+1+(m-1) i}\right\rangle_{\bar{p}} \\
& \oplus\left\langle e_{i}, \quad e_{2 i}, \quad e_{3 i}, \quad \ldots, \quad e_{m i}\right\rangle_{i-1} \text {. }
\end{aligned}
$$

(1.3) If $a=b$ and $a^{i} \neq 1,0<i<n$. We have the $\mathbb{Z}$-grading

$$
A=\left\langle e_{1}, e_{2}\right\rangle_{1} \oplus\left\langle e_{3}\right\rangle_{2} \oplus\left\langle e_{4}\right\rangle_{3} \oplus \cdots \oplus\left\langle e_{n-1}\right\rangle_{n-2} \oplus\left\langle e_{n}\right\rangle_{n-1} .
$$

(2) $a \neq b$. We have $d_{1}=a, d_{2}=b, d_{i}=a^{i-2} b$ with $3 \leq i \leq n$.

(2.1) If $a=1$. We obtain a $\mathbb{Z}_{2}$-grading

$$
A=\left\langle e_{1}\right\rangle_{\overline{0}} \oplus\left\langle e_{2}, e_{3}, \ldots, e_{n}\right\rangle_{\overline{1}} .
$$

(2.2) If $a=-1$ and $b=1$.

We get the $\mathbb{Z}_{2}$-grading

$$
A=\left\langle e_{2}, e_{4}, e_{6}, \ldots\right\rangle_{\overline{0}} \oplus\left\langle e_{1}, e_{3}, e_{5}, e_{7}, \ldots\right\rangle_{\overline{1}} .
$$

(2.3) If $a=-1$ and $b \neq 1$.

We get the $\mathbb{Z} \times \mathbb{Z}_{2}$-grading

$$
A=\left\langle e_{1}\right\rangle_{(0, \overline{1})} \oplus\left\langle e_{2}, e_{4}, e_{6}, \ldots\right\rangle_{(1, \overline{0})} \oplus\left\langle e_{3}, e_{5}, e_{7}, \ldots\right\rangle_{(1, \overline{1})} .
$$

(2.4) If $a \notin\{1,-1\}$.

(2.4.1) If $b=1$, then $d_{1}=a, d_{2}=1, d_{i}=a^{i-2}$ with $3 \leq i \leq n$. We can distinguish two cases: 
(A) If there exists $i$ with $3 \leq i \leq n-2$ such that $a^{i}=1$. Let $n=m i+p$, $0 \leq p \leq i-1$. We have the following $\mathbb{Z}_{i}$-grading

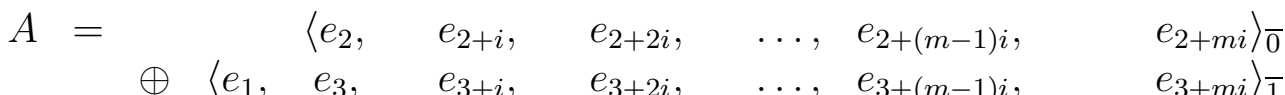

$$
\begin{aligned}
& \oplus \quad\left\langle e_{p}, \quad e_{p+i}, \quad e_{p+2 i}, \quad \ldots, \quad e_{p+(m-1) i}, \quad e_{p+m i}\right\rangle_{\overline{p-2}} \\
& \oplus \quad\left\langle e_{p+1}, \quad e_{p+1+i}, \quad e_{p+1+2 i}, \quad \ldots, \quad e_{p+1+(m-1) i}\right\rangle_{\overline{p-1}} \\
& \oplus \quad\left\langle e_{i+1}, \quad e_{2 i+1}, \quad e_{3 i+1}, \quad \ldots, \quad e_{i+1+(m-1) i}\right\rangle_{\overline{i-1}} .
\end{aligned}
$$

(B) If $a^{i} \neq 1$ for any $i, 3 \leq i \leq n-2$. We have the following $\mathbb{Z}$-grading:

$$
A=\left\langle e_{2}\right\rangle_{0} \oplus\left\langle e_{1}, e_{3}\right\rangle_{1} \oplus\left\langle e_{4}\right\rangle_{2} \oplus \cdots \oplus\left\langle e_{n-1}\right\rangle_{n-3} \oplus\left\langle e_{n}\right\rangle_{n-2} .
$$

(2.4.2) If $b \neq 1$.

(A) $d_{i} \neq d_{j}$ for all $i, j$ with $1 \leq i, j \leq n$. We have the following $\mathbb{Z}$-grading:

$$
A=\left\langle e_{1}\right\rangle_{1} \oplus\left\langle e_{2}\right\rangle_{2} \oplus\left\langle e_{3}\right\rangle_{3} \oplus\left\langle e_{4}\right\rangle_{4} \oplus \cdots \oplus\left\langle e_{n-1}\right\rangle_{n-1} \oplus\left\langle e_{n}\right\rangle_{n} .
$$

(B) there exist $k, l$ with $k \neq l$ such that $d_{k}=d_{l}$ with $3 \leq k<l \leq n$. Thus, $a^{i}=1$. Let $n=m i+p$ be. We have the following $\mathbb{Z} \times \mathbb{Z}_{i}$-grading:

$$
\begin{aligned}
& A=\left\langle e_{1}\right\rangle_{(0, \overline{1})} \\
& \oplus\left\langle e_{2}, \quad e_{2+i}, \quad e_{2+2 i}, \quad \ldots, \quad e_{2+(m-1) i}, \quad e_{2+m i}\right\rangle_{(1, \overline{0})} \\
& \oplus\left\langle e_{3}, \quad e_{3+i}, \quad e_{3+2 i}, \quad \ldots, \quad e_{3+(m-1) i}, \quad e_{3+m i}\right\rangle_{(1, \overline{1})} \\
& \oplus\left\langle e_{p}, \quad e_{p+i}, \quad e_{p+2 i}, \quad \ldots, \quad e_{p+(m-1) i}, \quad e_{p+m i}\right\rangle_{(1, \overline{p-2})} \\
& \oplus\left\langle e_{p+1}, \quad e_{p+1+i}, \quad e_{p+1+2 i}, \quad \ldots, \quad e_{p+1+(m-1) i}\right\rangle_{(1, \overline{p-1})} \\
& \oplus\left\langle e_{i+1}, \quad e_{2 i+1}, \quad e_{3 i+1}, \quad \ldots, \quad e_{i+1+(m-1) i}\right\rangle_{(1, \overline{i-1})} .
\end{aligned}
$$

(C) there exists $i, 3 \leq i \leq n$ such that $d_{1}=d_{i}$ and $d_{1} \neq d_{j}, i \neq j$. Thus, $b=\frac{1}{a^{i-3}}$. We put $a^{i-3} \neq 1$ because in other case $b=1$ and it gives the case (2.4.1.B). We have the following $\mathbb{Z}$-grading:

$$
A=\left\langle e_{2}\right\rangle_{-i+3} \oplus \cdots \oplus\left\langle e_{i-2}\right\rangle_{-1} \oplus\left\langle e_{i-1}\right\rangle_{0} \oplus\left\langle e_{1}, e_{i}\right\rangle_{1} \oplus\left\langle e_{i+1}\right\rangle_{2} \oplus \cdots \oplus\left\langle e_{n}\right\rangle_{n-i+1},
$$

(D) there exist $k, l$ with $k>l$ such that $d_{1}=d_{k}=d_{l}$ and $k-l=i$. Thus $b=a^{3-l}$ and $a^{i}=1$. Let $n=m i+p$ with $0 \leq p<i$ be. We have the following $\mathbb{Z}_{i}$-grading:

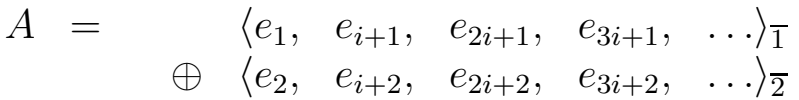

$$
\begin{aligned}
& \oplus\left\langle e_{i}, \quad e_{2 i}, \quad e_{3 i}, \quad e_{4 i}, \quad \ldots\right\rangle_{\overline{0}} .
\end{aligned}
$$

Since one-dimensional filiform Leibniz algebras and two-dimensional filiform Leibniz algebras with $\theta=0$ have zero product we consider filiform Leibniz algebras, with $\theta=0$, of dimension greater or equal than three.

Lemma 15. Let $A$ be a one-parametric filiform Leibniz algebra with $\theta=0$ and of dimension $n \geq 3$. Then any cyclic grading is equivalent to only one of the following: 
(1) The trivial grading given by $A=\left\langle e_{1}, e_{2}, \ldots, e_{n}\right\rangle$.

(2) The $\mathbb{Z}$-grading given by

$$
A=\left\langle e_{1}\right\rangle_{1} \oplus\left\langle e_{2}\right\rangle_{2} \oplus\left\langle e_{3}\right\rangle_{3} \oplus\left\langle e_{4}\right\rangle_{4} \oplus \cdots \oplus\left\langle e_{n-1}\right\rangle_{n-1} \oplus\left\langle e_{n}\right\rangle_{n} .
$$

(3) The $\mathbb{Z}$-grading given by

$$
A=\left\langle e_{1}, e_{2}\right\rangle_{1} \oplus\left\langle e_{3}\right\rangle_{2} \oplus\left\langle e_{4}\right\rangle_{3} \oplus \cdots \oplus\left\langle e_{n-1}\right\rangle_{n-2} \oplus\left\langle e_{n}\right\rangle_{n-1} .
$$

(4) For any $3 \leq i \leq n$, the $\mathbb{Z}$-grading given by

$$
A=\left\langle e_{2}\right\rangle_{-i+3} \oplus \cdots \oplus\left\langle e_{i-2}\right\rangle_{-1} \oplus\left\langle e_{i-1}\right\rangle_{0} \oplus\left\langle e_{1}, e_{i}\right\rangle_{1} \oplus\left\langle e_{i+1}\right\rangle_{2} \oplus \cdots \oplus\left\langle e_{n}\right\rangle_{n-i+1} .
$$

(5) The $\mathbb{Z}_{2}$-grading given by

$$
A=\left\langle e_{1}\right\rangle_{\overline{0}} \oplus\left\langle e_{2}, e_{3}, \ldots, e_{n}\right\rangle_{\overline{1}} .
$$

(6) For $n \geq 4$, the $\mathbb{Z}_{2}$-grading given by

$$
A=\left\langle e_{2}, e_{4}, e_{6}, \ldots\right\rangle_{\overline{0}} \oplus\left\langle e_{1}, e_{3}, e_{5}, e_{7}, \ldots\right\rangle_{\overline{1}} .
$$

(7) The $\mathbb{Z} \times \mathbb{Z}_{2}$-grading given by

$$
A=\left\langle e_{1}\right\rangle_{(0, \overline{1})} \oplus\left\langle e_{2}, e_{4}, e_{6}, \ldots\right\rangle_{(1, \overline{0})} \oplus\left\langle e_{3}, e_{5}, e_{7}, \ldots\right\rangle_{(1, \overline{1})} .
$$

(8) For any $2 \leq i \leq n-1,(i, n) \neq(2,3)$, the $\mathbb{Z}_{i}$-grading given by

$$
\begin{aligned}
& A=\quad\left\langle e_{i+1}, \quad e_{2 i+1}, \quad \ldots, \quad e_{1+(m-1) i}, \quad e_{1+m i}\right\rangle_{\overline{0}} \\
& \oplus\left\langle e_{1}, e_{2}, \quad e_{2+i}, \quad e_{2+2 i}, \quad \ldots, \quad e_{2+(m-1) i}, \quad e_{2+m i}\right\rangle_{\overline{1}} \\
& \oplus\left\langle e_{3}, \quad e_{3+i}, \quad e_{3+2 i}, \quad \ldots, \quad e_{3+(m-1) i}, \quad e_{3+m i}\right\rangle_{2} \\
& \oplus\left\langle e_{p}, \quad e_{p+i}, \quad e_{p+2 i}, \quad \ldots, \quad e_{p+(m-1) i}, \quad e_{p+m i}\right\rangle_{\overline{p-1}} \\
& \oplus\left\langle e_{p+1}, \quad e_{p+1+i}, \quad e_{p+1+2 i}, \quad \ldots, \quad e_{p+1+(m-1) i}\right\rangle_{\bar{p}} \\
& \oplus\left\langle e_{i}, \quad e_{2 i}, \quad e_{3 i}, \quad \ldots, \quad e_{m i}\right\rangle \overline{i-1} .
\end{aligned}
$$

(9) For any $3 \leq i \leq n-1,(i, n) \neq(3,4)$, the $\mathbb{Z}_{i}$-grading given by

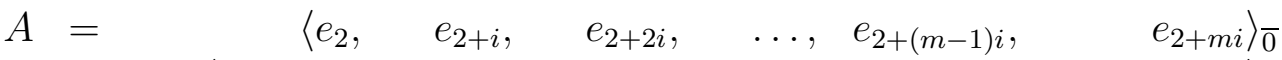

$$
\begin{aligned}
& \oplus\left\langle e_{1}, \quad e_{3}, \quad e_{3+i}, \quad e_{3+2 i}, \quad \ldots, \quad e_{3+(m-1) i}, \quad e_{3+m i}\right\rangle_{\overline{1}} \\
& \oplus \quad\left\langle e_{p}, \quad e_{p+i}, \quad e_{p+2 i}, \quad \ldots, \quad e_{p+(m-1) i}, \quad e_{p+m i}\right\rangle_{\overline{p-2}} \\
& \oplus \quad\left\langle e_{p+1}, \quad e_{p+1+i}, \quad e_{p+1+2 i}, \quad \ldots, \quad e_{p+1+(m-1) i}\right\rangle_{\overline{p-1}} \\
& \oplus \quad\left\langle e_{i+1}, \quad e_{2 i+1}, \quad e_{3 i+1}, \quad \ldots, \quad e_{i+1+(m-1) i}\right\rangle \overline{i-1} .
\end{aligned}
$$

(10) For any $3 \leq i \leq n-2$, the $\mathbb{Z} \times \mathbb{Z}_{i}$-grading given by

$$
\begin{aligned}
& A= \\
& =\left\langle e_{1}\right\rangle_{(0, \overline{1})} \\
& \oplus\left\langle e_{2}, \quad e_{2+i},\right. \\
& \oplus\left\langle e_{3}, \quad e_{3+i},\right. \\
& e_{2+2 i}, \quad \ldots, \quad e_{2+(m-1) i}, \\
& e_{3+2 i}, \quad \ldots, e_{3+(m-1) i}, \\
& \left.e_{2+m i}\right\rangle_{(1, \overline{0})} \\
& \text {... } \\
& e_{p+i}, \quad e_{p+2 i}, \quad \ldots, \quad e_{p+(m-1) i}, \\
& \oplus\left\langle e_{p}, \quad e_{p+i},\right. \\
& \oplus\left\langle e_{p+1}, \quad e_{p+1+i}, \quad e_{p+1+2 i}, \quad \ldots, \quad e_{p+1+(m-1) i}\right\rangle_{(1, \overline{p-1})} \\
& \oplus\left\langle e_{i+1}, \quad e_{2 i+1}, \quad e_{3 i+1}, \quad \ldots, \quad e_{i+1+(m-1) i}\right\rangle_{(1, \overline{i-1})} . \\
& \left.e_{3+m i}\right\rangle_{(1, \overline{1})} \\
& \left.e_{p+m i}\right\rangle_{(1, \overline{p-2})}
\end{aligned}
$$


(11) For any $3 \leq i \leq n-2$, the $\mathbb{Z}_{i}$-grading given by

$$
\begin{aligned}
& A=\quad\left\langle e_{1}, \quad e_{i+1}, \quad e_{2 i+1}, \quad e_{3 i+1}, \quad \ldots\right\rangle_{\overline{1}} \\
& \oplus\left\langle e_{2}, \quad e_{i+2}, \quad e_{2 i+2}, \quad e_{3 i+2}, \quad \ldots\right\rangle_{\overline{2}} \\
& \text {... } \\
& \oplus\left\langle e_{i}, \quad e_{2 i}, \quad e_{3 i}, \quad e_{4 i}, \quad \ldots\right\rangle_{\overline{0}} .
\end{aligned}
$$

Proof. By the above discussion, we just have to prove that two different gradings of the lemma are not equivalent.

Since two gradings with supports of different cardinal are not equivalent, we have that (1), (2) and (3) are not equivalent among them.

Consider now the gradings in the family, depending on $i$, (4) having all of them a support with cardinal $n-1$. From here these are not equivalent to (1) or (2). Clearly the grading (3) neither is equivalent to any grading in (4), because in the opposite case there should be an automorphism $\phi$ of $A$ such that $\phi\left(\left\langle e_{1}, e_{2}\right\rangle\right)=\left\langle e_{1}, e_{i}\right\rangle$ with $i \geq 3$, what would be a contradiction. If we fix now $3 \leq i, j \leq n$ with $i \neq j$ and consider the two gradings of type (4) associated to $i$ and $j$. In case both gradings were equivalent, we would have an automorphism $\phi$ of $A$ such that $\phi\left(\left\langle e_{1}, e_{i}\right\rangle\right)=\left\langle e_{1}, e_{j}\right\rangle$, but the dimension of the subalgebra of $A$ generated by $\left\{e_{1}, e_{i}\right\}$ is $n-i+2$ while the one generated by $\left\{e_{1}, e_{j}\right\}$ is $n-j+2$. Hence there not exists such $\phi$ because $i \neq j$. We conclude that two different gradings of the family (4) are not equivalent.

Consider now the grading (5). This is not equivalent to any of the previous ones because of the different cardinals of the supports, (in the case (3) when $n=3$ as consequence of there is not any automorphism satisfying $\phi\left(\left\langle e_{1}, e_{2}\right\rangle\right)=\left\langle e_{2}, e_{3}\right\rangle$ while in the case (4) when $n=3$ because there is not any automorphism satisfying $\left.\phi\left(\left\langle e_{1}, e_{3}\right\rangle\right)=\left\langle e_{2}, e_{3}\right\rangle\right)$.

As above, the grading (6) is not equivalent to any grading (1)-(5).

Respect to the grading (7), we get as above that this is not equivalent to (1)-(6).

Taking into account that the dimension of the homogeneous subspace containing $e_{1}$ is at least three in grading (8), we have as above that grading (8) is not equivalent to (1)-(7).

We observe that any grading in (9) has at least two homogeneous components with dimensions greater or equal that two, so they are not equivalent to any grading (1)-(7).

Since in (8) any grading has $e_{1}$ and $e_{2}$ in the same homogeneous component, we also have that (9) is not equivalent to (8).

Taking now into account that in gradings (10) there is always at least four nonzero homogeneous components, that $\left\langle e_{1}\right\rangle$ is always a homogeneous component and that there exists at least one homogeneous component with dimension greater o equal than two, we get that gradings in (10) are not equivalent to any grading in (1)-(9).

Finally, by observing that in (11) all of the gradings have at least two homogeneous components with dimensions greater or equal than two, that $e_{1}$ does not belong to a one-dimensional homogeneous component, that $e_{2}$ does not belong to the same component than $e_{1}$ and that $e_{3}$ belongs to the same component than $e_{1}$ just in case $i=2$, we can assert that any grading in (11) is not equivalent to any grading in (1)-(10).

Next, we are going to show that any abelian group grading on $A$ is equivalent to a cyclic grading.

Lemma 16. If $e_{1}, e_{2}$ are homogeneous elements of an abelian group grading of $A$ (that is, $e_{1} \in$ $A_{x}, e_{2} \in A_{y}$ for some $\left.x, y \in G\right)$, then the grading of $A$ is one of the list in Lemma 15 
Proof. Let $e_{1} \in A_{x}, e_{2} \in A_{y}$ and $x, y \in G$ be; and let denote by $i$ be the order of $x$ (denoted by $\operatorname{ord}(x)=i$ ). We are going to distinguish two cases.

In the first one $x=y$. That is $e_{1}, e_{2} \in A_{x}$. We can consider the next possibilities:

- We have $i \geq n$. We get by the grading $e_{3}=e_{2} e_{1} \in A_{2 x}, e_{4}=e_{3} e_{1} \in A_{3 x}$ and so $e_{j}=$ $e_{j-1} e_{1} \in A_{(j-1) x}$ for any $j \leq n$. From here we have the grading

$$
A=\left\langle e_{1}, e_{2}\right\rangle_{x} \oplus\left\langle e_{3}\right\rangle_{2 x} \oplus\left\langle e_{4}\right\rangle_{3 x} \oplus \cdots \oplus\left\langle e_{n-1}\right\rangle_{(n-2) x} \oplus\left\langle e_{n}\right\rangle_{(n-1) x},
$$

which is clearly equivalent to the $\mathbb{Z}$-grading in Lemma 15 -(3).

- We have $1 \leq i \leq n-1$. As above we get that $e_{j} \in A_{(j-1) x}$ for $1 \geq j \geq n$. Hence, in case $i=1$ (and so $x=0$ ) we get that $A$ has the trivial grading. Suppose then $2 \leq i \leq n-1$. Then

$$
e_{1}, e_{2} \in A_{x}, e_{3} \in A_{2 x}, \ldots, e_{i} \in A_{(i-1) x} \text { with }|\{x, 2 x, \ldots,(i-1) x\}|=i-1,
$$

and also

$$
e_{i}=e_{i-1} e_{1} \in A_{i x}=A_{0}
$$

because ord $(x)=i$.

Finally, let $j \geq i+1$ be. Since $j=i r+s$ with $r, s \in \mathbb{N}$ and $0 \leq s<i$, we get as consequence of $\operatorname{ord}(x)=i$ that

$$
e_{j} \in A_{j x}=-A_{s x}
$$

From here, if we write $n=i m+p$ with $m, p \in \mathbb{N}$ and $0 \leq p<i$, we have the grading

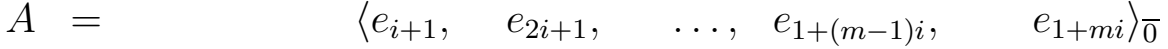

$$
\begin{aligned}
& \oplus\left\langle e_{1}, e_{2}, \quad e_{2+i}, \quad e_{2+2 i}, \quad \ldots, \quad e_{2+(m-1) i}, \quad e_{2+m i}\right\rangle_{\overline{1}} \\
& \oplus\left\langle e_{3}, \quad e_{3+i}, \quad e_{3+2 i}, \quad \ldots, \quad e_{3+(m-1) i}, \quad e_{3+m i}\right\rangle_{\overline{2}} \\
& \oplus\left\langle e_{p}, \quad e_{p+i}, \quad e_{p+2 i}, \quad \ldots, \quad e_{p+(m-1) i}, \quad e_{p+m i}\right\rangle_{\overline{p-1}} \\
& \oplus\left\langle e_{p+1}, \quad e_{p+1+i}, \quad e_{p+1+2 i}, \quad \ldots, \quad e_{p+1+(m-1) i}\right\rangle_{\bar{p}} \\
& \oplus\left\langle e_{i}, \quad e_{2 i}, \quad e_{3 i}, \quad \ldots, \quad e_{m i}\right\rangle \overline{i-1} \text {. }
\end{aligned}
$$

This grading is equivalent to the $\mathbb{Z}$-grading in Lemma 15 -(3) when $(i, n)=(2,3)$ or to the $\mathbb{Z}_{i}$-grading in Lemma15-(8) when $(i, n) \neq(2,3)$.

In the second case $x \neq y$. That is $e_{1} \in A_{x}, e_{2} \in A_{y}$ with $x \neq y$. We distinguish three possibilities.

- First $i=1$. Then $x=0$ and so

$$
e_{j}=\left(\ldots \left((e_{2} \underbrace{\left.\left.\left.e_{1}\right) e_{1}\right) \ldots\right) e_{1}}_{j-2} \in A_{y}\right.\right.
$$

for any $3 \leq j \leq n$. Hence

$$
A=\left\langle e_{1}\right\rangle_{0} \oplus\left\langle e_{2}, e_{3}, \ldots, e_{n}\right\rangle_{y},
$$

which is equivalent to the $\mathbb{Z}_{2}$-grading in Lemma 15 -(5).

- Second, $i \geq n-1$. We have as above that

$$
e_{j} \in A_{y+(j-2) x}
$$

for any $3 \leq j \leq n$. Observe that the fact $\operatorname{ord}(x) \geq n-1$ gives us that $y+p x \neq y+q x$ and $y+p x \neq y$ for any $1 \leq p, q \leq n-2$ with $p \neq q$. However, it is possible that $y+p x=x$ for some $1 \leq p \leq n-2$. That is, $y=-k x$ for some $0 \leq k \leq n-3$. Form here, we have that to distinguish two cases so as to obtain: 
If $y \neq-k x$ for any $k \in\{0,1, \ldots, n-3\}$ we get the grading

$$
A=\left\langle e_{1}\right\rangle_{x} \oplus\left\langle e_{2}\right\rangle_{y} \oplus\left\langle e_{3}\right\rangle_{y+x} \oplus\left\langle e_{4}\right\rangle_{y+2 x} \oplus \cdots \oplus\left\langle e_{n-1}\right\rangle_{y+(n-3) x} \oplus\left\langle e_{n}\right\rangle_{y+(n-2) x},
$$

which is equivalent to the $\mathbb{Z}$-grading in Lemma 15 -(2).

If $y=-k x$ for some $k \in\{0,1, \ldots, n-3\}$ then we have

$$
\begin{aligned}
A= & \left\langle e_{2}\right\rangle_{-k x} \oplus\left\langle e_{3}\right\rangle_{(-k+1) x} \oplus \cdots \oplus\left\langle e_{k+1}\right\rangle_{-x} \oplus\left\langle e_{k+2}\right\rangle_{0} \oplus\left\langle e_{1}, e_{k+3}\right\rangle_{x} \oplus \\
& \oplus\left\langle e_{k+4}\right\rangle_{2 x} \oplus \cdots \oplus\left\langle e_{n}\right\rangle_{(n-k-2) x},
\end{aligned}
$$

which is equivalent to the $\mathbb{Z}$-grading in Lemma 15 -(4).

- Third, $2 \leq i \leq n-2$. Then,

$e_{1} \in A_{x}, e_{2} \in A_{y}, e_{3} \in A_{y+x}, \ldots, e_{i+1} \in A_{y+(i-1) x}$ with $|\{x, 2 x, \ldots,(i-1) x\}|=i-1$.

We also have

$$
e_{i+2} \in A_{y+i x}=A_{y} .
$$

Take now some $j \geq i+3$ and express $j-2=i r+s$ with $r, s \in \mathbb{N}$ and $0 \leq s<i$, then

$$
e_{j} \in A_{y+(j-2) x}=A_{s} .
$$

Now if we express $n=i m+p$ with $m, p \in \mathbb{N}$ and $0 \leq p<i$, we can distinguish as above two possibilities.

In the first one $y \neq-k x$ for any $k \in\{0,1, \ldots, n-3\}$. Then we get the grading

$$
\begin{aligned}
& A=\left\langle e_{1}\right\rangle_{x} \\
& \oplus\left\langle e_{2}, \quad e_{2+i}, \quad e_{2+2 i}, \quad \ldots, \quad e_{2+(m-1) i}, \quad e_{2+m i}\right\rangle_{y} \\
& \oplus\left\langle e_{3}, \quad e_{3+i}, \quad e_{3+2 i}, \quad \ldots, \quad e_{3+(m-1) i}, \quad e_{3+m i}\right\rangle_{y+x} \\
& \left\langle e_{p}, \quad e_{p+i}, \quad e_{p+2 i}, \quad \ldots, \quad e_{p+(m-1) i}, \quad e_{p+m i}\right\rangle_{y+(p-2) x} \\
& \oplus\left\langle e_{p+1}, \quad e_{p+1+i}, \quad e_{p+1+2 i}, \ldots, \quad e_{p+1+(m-1) i}\right\rangle_{y+(p-1) x} \\
& \text {... } \\
& \oplus\left\langle e_{i+1}, \quad e_{2 i+1}, \quad e_{3 i+1}, \quad \ldots, \quad e_{i+1+(m-1) i}\right\rangle_{y+(i-1) x},
\end{aligned}
$$

which is equivalent to the $\mathbb{Z} \times \mathbb{Z}_{2}$-grading in Lemma 15 -(7) when $i=2$ or to $\mathbb{Z} \times \mathbb{Z}_{i}$-grading in Lemma 15-(10) when $i \geq 3$.

In the second one $y=-k x$ for some $k \in\{0,1, \ldots, n-3\}$. Then we have the grading

$$
\begin{aligned}
& A=\quad \oplus \begin{array}{ccccc}
\left\langle e_{1},\right. & e_{k+3}, & e_{k+3+i}, & e_{k+3+2 i}, & \ldots\rangle_{x} \\
\left\langle e_{2},\right. & e_{k+4}, & e_{k+4+i}, & e_{k+4+2 i}, & \ldots\rangle_{2 x}
\end{array} \\
& \oplus\left\langle e_{k+1}, \quad e_{k+1+i}, \quad e_{k+1+2 i}, \quad e_{k+1+3 i}, \quad \ldots\right\rangle_{(i-1) x} \\
& \oplus\left\langle e_{k+2}, \quad e_{k+2+i}, \quad e_{k+2+2 i}, \quad e_{k+2+3 i}, \quad \ldots\right\rangle_{0} \text {. }
\end{aligned}
$$

which is equivalent either to the $\mathbb{Z}_{2}$-grading in 15 -(6) when $k=0$ (that is, $y=0$ ) and $i=2$ or to the $\mathbb{Z}_{i}$-grading in 15 -(9) when $k=0$ and $i \geq 3$, or to the $\mathbb{Z} \times \mathbb{Z}_{2}$-grading in Lemma 15-(7) when $k \neq 0$ (that is, $y \neq 0$ ) and $i=2$ or to the $\mathbb{Z}_{i}$-grading in 15 -(11) when $k \neq 0$ and $i \geq 3$.

Theorem 17. Any group grading of a one-parametric filiform Leibniz algebra with $\theta=0$, and dimension $n \geq 3$, is equivalent to only one in the list of Lemma 15 
Proof. Recall that any grading of $A$ is induced by an abelian subgroup $\mathcal{G}$ of diagonalizable automorphisms in $\mathcal{N}(\mathcal{T})=\mathcal{T}$, being the homogeneous components of the grading the common eigenspaces of the elements in $\mathcal{G}$.

Since for any $f \in \mathcal{G} \subset \mathcal{T}$, Equation (3) gives us that $e_{1}$ and $e_{2}$ are eigenvectors of $f$, then $e_{1}$ and $e_{2}$ are homogeneous vectors in any grading of $A$. From here Lemma 16 completes the proof.

4.3. Case in which $\theta \neq 0$. Taking into account Equation (2), we have that in case $\operatorname{dim}\left(F_{1}\right) \geq 4$ then

$$
\operatorname{Aut}\left(F_{1}\right)=\left\{\left(\begin{array}{lllll}
\epsilon & 0 & 0 & \ldots & 0 \\
0 & b & 0 & \ldots & 0 \\
0 & b_{3} & \epsilon b & \ldots & 0 \\
0 & b_{4} & \epsilon b_{3} & \ldots & 0 \\
\vdots & \vdots & \vdots & \ddots & \vdots \\
a_{n} & b_{n} & \epsilon b_{n-1} & \ldots & \epsilon^{n-2} b
\end{array}\right): a_{n}, b_{3}, \ldots, b_{n} \in \mathbb{C}, b \in \mathbb{C}^{*} ; \epsilon^{n-3}=1\right\}
$$

From here a maximal torus is:

$$
\mathcal{T}=\left\{t_{1, b}:=\left(\begin{array}{ccccc}
1 & 0 & 0 & \ldots & 0 \\
0 & b & 0 & \ldots & 0 \\
0 & 0 & b & \ldots & 0 \\
\vdots & \vdots & \vdots & \ddots & \vdots \\
0 & 0 & 0 & \ldots & b
\end{array}\right): b \in \mathbb{C}^{*}\right\} \cong \mathbb{C}^{*}
$$

Hence, the normalizer of $\mathcal{T}$ in $\operatorname{Aut}\left(F_{1}\right)$ is

$$
\mathcal{N}(\mathcal{T})=\left\{\left(\begin{array}{lllll}
\epsilon & 0 & 0 & \ldots & 0 \\
0 & b & 0 & \ldots & 0 \\
0 & b_{3} & \epsilon b & \ldots & 0 \\
0 & b_{4} & \epsilon b_{3} & \ldots & 0 \\
\vdots & \vdots & \vdots & \ddots & \vdots \\
0 & b_{n} & \epsilon b_{n-1} & \ldots & \epsilon^{n-2} b
\end{array}\right): b_{3}, \ldots, b_{n} \in \mathbb{C}, b \in \mathbb{C}^{*} ; \epsilon^{n-3}=1\right\}
$$

Observe that $\mathcal{T} \varsubsetneqq \mathcal{N}(\mathcal{T})$ and so any grading of $F_{1}$ is not necessarily a toral one.

Let us denote by $A$ any (non-Lie) one-parametric filiform Leibniz with $\theta \neq 0$ and with $\operatorname{dim}(A) \geq 3$ (the cases $\operatorname{dim}(A)=1,2$ give algebras with zero product).

Recall now that any grading of $A$ is induced by an abelian subgroup $\mathcal{G}$ of diagonalizable automorphisms in $\mathcal{N}(\mathcal{T})$, being the homogeneous components of the grading the common eigenspaces of the elements in $\mathcal{G}$.

Since for any $f \in \mathcal{G}$ (and following from the multiplication table of the algebra), $e_{1}$ and $e_{n}$ are eigenvectors of $f$, then $e_{1}$ and $e_{n}$ are homogeneous vectors in any grading of $A$. That is,

$$
e_{1} \in A_{g} \text { and } e_{n} \in A_{h}
$$

for some $g, h \in G$, where $G$ denotes the abelian group in the grading of $A$ :

$$
A=\bigoplus_{g \in G} A_{g}
$$

If $A$ is three-dimensional, we know by Equation (4) that $e_{1} \in A_{g}$ and $e_{3} \in A_{h}$. We are going to distinguish two possibilities: 
First, $g=h$. In this case either $A=A_{g}$ when $e_{2} \in A_{g}$ or $A=A_{g} \oplus A_{t}$ with $t \neq g$ when $e_{2} \notin A_{g}$. In the first case we get the trivial grading $A=A_{0}$. In the second case we can write $e_{2}=v_{g}+v_{t}$ with $v_{g} \in A_{g}$ and $0 \neq v_{t} \in A_{t}$. If $v_{g}=0$ then $e_{2} \in A_{t}$ and we have the $\mathbb{Z}_{2}$-grading

$$
A=\left\langle e_{2}\right\rangle_{\overline{0}} \oplus\left\langle e_{1}, e_{3}\right\rangle_{\overline{1}} .
$$

If $v_{g} \neq 0$ then the fact $e_{1} e_{2}=\theta e_{3}$ and the grading of $A$ give us $2 g=g$ and $g+t=g$. From here $g=0$ and so $t=0$. Hence $g=t$, a contradiction, and so this case does not happen.

Second, $g \neq h$. In this case either $A=A_{g} \oplus A_{h}$ when $e_{2} \in A_{g} \oplus A_{h}$ or $A=A_{g} \oplus A_{h} \oplus A_{t}$ with $t \notin\{g, h\}$ when $e_{2} \notin A_{g} \oplus A_{h}$.

Consider the case in which $e_{2} \in A_{g} \oplus A_{h}$ and write $e_{2}=v_{g}+v_{h}$ with $v_{g} \in A_{g}$ and $v_{h} \in A_{h}$. If $v_{g}=0$ we get $e_{2}=e_{h}$ and the the $\mathbb{Z}_{2}$-grading

$$
A=\left\langle e_{1}\right\rangle_{\overline{0}} \oplus\left\langle e_{2}, e_{3}\right\rangle_{\overline{1}} .
$$

If $v_{h}=0$ then $e_{2}=v_{h}$ and we obtain the the $\mathbb{Z}_{2}$-grading

$$
A=\left\langle e_{3}\right\rangle_{\overline{0}} \oplus\left\langle e_{1}, e_{2}\right\rangle_{\overline{1}} .
$$

Finally if $v_{g} \neq 0$ and $v_{h} \neq 0$, the fact $e_{1} e_{2}=\theta e_{3}$ and the grading of $A$ give us $2 g=h$ and $g+h=h$. From here $g=h=0$. This is a contradiction and so this case does not happen.

Consider now the case in which $e_{2} \notin A_{g} \oplus A_{h}$. Then we can write $e_{2}=v_{g}+v_{h}+v_{t}$ with $v_{i} \in A_{i}$ for $i \in\{g, h, t\}$ and $v_{t} \neq 0$. If $v_{g}=v_{h}=0$ then $e_{2}=v_{t}$ and we get the $\mathbb{Z}$-grading

$$
A=\left\langle e_{1}\right\rangle_{1} \oplus\left\langle e_{2}\right\rangle_{2} \oplus\left\langle e_{2}\right\rangle_{3} \text {. }
$$

If $v_{g} \neq 0$ we get as above that $2 g=h$ and that $g+t=h$. From here $t=g$ a contradiction. If $v_{h} \neq 0$ we have in a similar way $g+h=h$ and $g+t=h$. From here $h=t$. A contradiction. Hence these last cases do not occur.

Since the grading (9) has three homogeneous components while the remaining non-trivial gradings have only two homogeneous components we have that the grading (9)is not equivalent to any grading in (6)-(8). The grading (6) is not equivalent to grading (8). Indeed, in the opposite case we would have an automorphism $\phi$ of $A$ satisfying $\phi\left(\left\langle e_{2}\right\rangle\right)=\left\langle e_{3}\right\rangle$ and $\phi\left(\left\langle e_{1}, e_{3}\right\rangle\right)=\left\langle e_{2}, e_{3}\right\rangle$. But in this case $0 \neq \phi\left(e_{3}\right)=\phi\left(e_{2}\right) \phi\left(e_{1}\right)=0$, a contradiction. In a similar way, gradings (7) and (8) are not equivalent. Finally, an analogous argument gives us that gradings (6) and (7) are equivalent if and only if $\theta \in\{ \pm 1\}$.

Let us summarize our results in the following statement.

Proposition 18. Any abelian group grading of a one-parametric filiform Leibniz algebra A (with a nonzero parameter) of dimension 3 is equivalent to the trivial grading or to one of the gradings (6), (7), (8) or (9) if $\theta \notin\{ \pm 1\}$; or to the trivial grading or to one of the gradings (6), (8) or (9) if $\theta \in\{ \pm 1\}$.

Below we will consider the case of the algebra $A$ for $n \geq 4$.

Now, since $e_{1} e_{2}=\theta e_{n}$ with $\theta \neq 0$, we can write by Equation (5) that

$$
e_{2}=v_{g_{1}}+v_{g_{2}}+\cdots+v_{g_{m}}
$$

with any $v_{g_{i}} \neq 0$ and $g_{i} \neq g_{j}$ when $i \neq j$. From here $e_{1}\left(v_{g_{1}}+v_{g_{2}}+\cdots+v_{g_{m}}\right)=\theta e_{n}$ and, by distinguish removing the zero and the non-zero products, we can write

$$
\sum_{j \in J} e_{1} v_{g_{j}}+\sum_{k \in K} e_{1} v_{g_{k}}=\theta e_{n}, J \cap K=\emptyset,
$$


with $e_{1} v_{g_{j}}=0$ for any $j \in J$ and $e_{1} v_{g_{k}} \neq 0$ for any $k \in K$. Since $e_{1} \in A_{g}$, then the fact $\sum_{k \in K} e_{1} v_{g_{k}}=$ $\theta e_{n}$ implies that by writing for any $k \in K, 0 \neq e_{1} v_{g_{k}}=v_{g_{k}+g} \in A_{g_{k}+g}$, we have that $0 \neq \sum_{k \in K} v_{g_{k}+g}=$ $\theta e_{n} \in A_{h}$. Hence by the grading, we get that $g_{k}+g=h$ for any $k \in K$. That is, $g_{k}=h-g$ for any $k \in K$. From here we have that the cardinal of $K$ is necessarily 1 and we write

$$
e_{2}=\sum_{j \in J} v_{g_{j}}+v_{h-g}
$$

where any $v_{g_{j}} \in A_{g_{j}}$ with $e_{1} v_{g_{j}}=0$ and $0 \neq v_{h-g} \in A_{h-g}$ with $e_{1} v_{h-g} \neq 0$.

Now, since $e_{2} e_{1}=e_{3}$, we have $\sum_{j \in J} v_{g_{j}} e_{1}+v_{h-g} e_{1}=e_{3}$. In case $v_{h-g} e_{1}=0$, then by the product in $A$ we get $0 \neq v_{h-g}=\alpha e_{1}+\beta e_{n}, \alpha, \beta \in \mathbb{C}$. But $v_{h-g}=e_{2}-\sum_{j \in J} v_{g_{j}}$ and so $e_{1} v_{h-g}=e_{1} e_{2}=e_{3} \neq 0$. However in this case $e_{1} v_{h-g}=e_{1}\left(\alpha e_{1}+\beta e_{n}\right)=0$ which is a contradiction (recall $\left.n \geq 4\right)$. Hence $v_{h-g} e_{1} \neq 0$ and we can write $0 \neq v_{h-g} e_{1}:=v_{h} \in A_{h}$. We get

$$
e_{3}=\sum_{j \in J} v_{g_{j}+g}+v_{h}
$$

with $v_{h} \neq 0$.

We have that $v_{h} e_{1} \neq 0$. Indeed, if $v_{h} e_{1}=0$ then $v_{h}=\alpha e_{1}+\beta e_{n}$ with $\alpha \neq 0$ or $\beta \neq 0$. But $0 \neq$ $v_{h}=v_{h-g} e_{1}$ and so (by the product in $A$ ), $\alpha=0, \beta \neq 0$ and $v_{h-g}=\tau e_{1}+\beta e_{n-1}+\gamma e_{n}$. Recall from the above that $e_{1} v_{h-g} \neq 0$ but $e_{1}\left(\tau e_{1}+\beta e_{n-1}+\gamma e_{n}\right)=0$, a contradiction. From here $v_{h} e_{1} \neq 0$ and by denoting $0 \neq v_{h} e_{1}:=v_{h+g} \in A_{h+g}$ we have that $e_{4}=e_{3} e_{1}=\left(\sum_{j \in J} v_{g_{j}+g}+v_{h}\right) e_{1}=\sum_{j \in J} v_{g_{j}+2 g}+v_{h+g}$ with $v_{h+g} \neq 0$. That is, we can assert

$$
e_{4}=\sum_{j \in J} v_{g_{j}+2 g}+v_{h+g}
$$

with $v_{h+g} \neq 0$.

By arguing in this way get for any $k \in\{2, \ldots, n-1\}$ that

$$
e_{k}=\sum_{j \in J} v_{g_{j}+(k-2) g}+v_{h+(k-3) g}
$$

with $v_{h+(k-3) g} \neq 0$.

Finally, for $k=n$ we have that the fact $e_{n-1} e_{1}=e_{n}$ gives us $e_{n}=e_{k}=\sum_{j \in J} v_{g_{j}+(n-2) g}+v_{h+(n-3) g}$ with $v_{h+(n-3) g} \neq 0$ and $e_{n} \in A_{h}$. From here, we get by the grading that $h+(n-3) g=h$ and so (recall $n \geq 4$ ),

$$
(n-3) g=0 .
$$

We have by the above that $(n-2) g=g$. So in case some $v_{g_{j}+(n-2) g} \neq 0$ we have $v_{g_{j}+(n-2) g} \in$ $A_{g_{j}+g}=A_{h}$ and so $g_{j}=h-g$. Summarizing we have (take into account that the fact $(n-3) g=0$ implies $(n-4) g=-g)$.

$$
\begin{gathered}
e_{1} \in A_{g}, \quad e_{2} \in A_{h-g}, \quad e_{3} \in A_{h}, \quad e_{4} \in A_{h+g}, \cdots, e_{k} \in A_{h+(k-3) g}, \cdots, e_{n-2} \in A_{h+(n-5) g}, \\
e_{n-1} \in A_{h+(n-4) g}=A_{h-g}, \quad e_{n} \in A_{h} .
\end{gathered}
$$

Let us distinguish two cases:

First, $g=0$. Then, by Equation (11), we have either the trivial grading

$$
A=A_{0}
$$


in case $h=0$ or the $\mathbb{Z}_{2}$-grading

$$
A=\left\langle e_{1}\right\rangle_{\overline{0}} \oplus\left\langle e_{2}, e_{3}, \ldots, e_{n}\right\rangle_{\overline{1}}
$$

when $h \neq 0$.

Second, $g \neq 0$. Observe that $\operatorname{dim}(A) \neq 4$ since in this case Equation (10) would give us $g=0$.

Let us denote by $2 \leq t \leq n-3$ the order of $g$. By Equation (10), $t$ divides $n-3$ and so we can write

$$
n-3=r t
$$

with $t, r \in \mathbb{N}$ being $2 \leq t \leq n-3$ and $1 \leq r \leq n-4$.

If furthermore $h=g$, Equation (11) gives us the following grading, which will be denote as

$$
\begin{aligned}
A_{g}=\left\langle e_{1}\right\rangle \oplus\left\langle e_{3}, e_{t+3}, e_{2 t+3}, \ldots, e_{(r-1) t+3}\right\rangle \oplus\left\langle e_{n}\right\rangle \\
A_{2 g}=\left\langle e_{4}, e_{t+4}, e_{2 t+4}, \ldots, e_{(r-1) t+4}\right\rangle \\
A_{3 g}=\left\langle e_{5}, e_{t+5}, e_{2 t+5}, \ldots, e_{(r-1) t+5}\right\rangle \\
\vdots \\
A_{(t-1) g}=\left\langle e_{t+1}, e_{2 t+1}, e_{3 t+1}, \ldots, e_{r t+1}\right\rangle \\
A_{0}=\left\langle e_{t+2}, e_{2 t+2}, e_{3 t+2}, \ldots, e_{r t+2}\right\rangle \oplus\left\langle e_{2}\right\rangle
\end{aligned}
$$

From here, for any divisor $t \neq 1$ of $n-3$ we have a $\mathbb{Z}_{t}$-grading of $A$ by taking $\bar{i}:=i g, i \in$ $\{0, \ldots, t-1\}$ in Equation (14) and where $r t=n-3$.

We note that $p g \neq q g$ for $p, q \in\{0, \ldots, t-1\}, p \neq q$, in Equation (14) since in the opposite case $(p-q) g=0$ with $p-q \leq t-1$, a contradiction with the order $t$ of $g$.

We also note that for $t=2$, Equation (14) means the $\mathbb{Z}_{2}$-grading of $A$ :

$$
\begin{aligned}
& A_{\overline{0}}=\left\langle e_{4}, e_{6}, e_{8}, \ldots, e_{2(r+1)}\right\rangle \oplus\left\langle e_{2}\right\rangle \\
& A_{\overline{1}}=\left\langle e_{1}\right\rangle \oplus\left\langle e_{3}, e_{5}, e_{7}, \ldots, e_{2 r+1}\right\rangle \oplus\left\langle e_{n}\right\rangle .
\end{aligned}
$$

It remains to study the case in which $h \neq g$ (and also $g \neq 0$ ). In this case Equation (11) gives us

$$
\begin{gathered}
A_{g}=\left\langle e_{1}\right\rangle \\
A_{h}=\left\langle e_{3}, e_{t+3}, e_{2 t+3}, \ldots, e_{(r-1) t+3}\right\rangle \oplus\left\langle e_{n}\right\rangle \\
A_{h+g}=\left\langle e_{4}, e_{t+4}, e_{2 t+4}, \ldots, e_{(r-1) t+4}\right\rangle \\
A_{h+2 g}=\left\langle e_{5}, e_{t+5}, e_{2 t+5}, \ldots, e_{(r-1) t+5}\right\rangle \\
\vdots \\
A_{h+(t-2) g}=\left\langle e_{t+1}, e_{2 t+1}, e_{3 t+1}, \ldots, e_{r t+1}\right\rangle \\
A_{h+(t-1) g}=\left\langle e_{2}\right\rangle \oplus\left\langle e_{t+2}, e_{2 t+2}, e_{3 t+2}, \ldots, e_{r t+2}\right\rangle .
\end{gathered}
$$

We have as above that $h+p g \neq h+q g$ for $p, q \in\{0, \ldots, t-1\}$. However it is possible that $g=h+p g$ for some $p \in\{1, \ldots, t-1\}$. Hence we consider two possibilities:

First, $g \neq h+p g$ for any $p \in\{1, \ldots, t-1\}$. Then we get that for any divisor $t \neq 1$ of $n-3$ we have a $\mathbb{Z}_{t} \times \mathbb{Z}$-grading of $A$ given by Equation (15) where $(\overline{1}, 0):=g,(\bar{i}, 1):=h+i g$ for $i \in\{0, \ldots, t-1\}$, and where $r t=n-3$.

We note that for $t=2$, Equation (15) means the $\mathbb{Z}_{2} \times \mathbb{Z}$-grading of $A$ :

$$
A_{(\overline{1}, 0)}=\left\langle e_{1}\right\rangle
$$




$$
\begin{aligned}
& A_{(\overline{0}, 1)}=\left\langle e_{3}, e_{5}, e_{7}, \ldots, e_{2 r+1}\right\rangle \oplus\left\langle e_{n}\right\rangle \\
& A_{(\overline{1}, 1)}=\left\langle e_{2}\right\rangle \oplus\left\langle e_{4}, e_{6}, e_{8}, \ldots, e_{2(r+1)}\right\rangle
\end{aligned}
$$

Second, $g=h+p g$ for some $p \in\{1, \ldots, t-1\}$. This fact is equivalent to $h=-\tau g$ for some $\tau \in\{0, \ldots, t-2\}$. In this case

$$
A_{g}=A_{h+(\tau+1) g}
$$

and, by looking at Equation (15), we obtain the grading

$$
\begin{aligned}
& A_{g}=\left\langle e_{1}\right\rangle \oplus\left\langle e_{\tau+4}, e_{t+\tau+4}, \ldots, e_{(r-1) t+\tau+4}\right\rangle \\
& A_{-\tau g}=\left\langle e_{3}, e_{t+3}, e_{2 t+3}, \ldots, e_{(r-1) t+3}\right\rangle \oplus\left\langle e_{n}\right\rangle \\
& A_{(-\tau+1) g}=\left\langle e_{4}, e_{t+4}, e_{2 t+4}, \ldots, e_{(r-1) t+4}\right\rangle \\
& A_{(-\tau+2) g}=\left\langle e_{5}, e_{t+5}, e_{2 t+5}, \ldots, e_{(r-1) t+5}\right\rangle \\
& \vdots \\
& A_{0}=\left\langle e_{\tau+3}, e_{t+\tau+3}, \ldots, e_{(r-1) t+\tau+3}\right\rangle \\
& \vdots \\
& A_{(t-\tau-2) g}=\left\langle e_{t+1}, e_{2 t+1}, e_{3 t+1}, \ldots, e_{r t+1}\right\rangle \\
& A_{(t-\tau-1) g}=\left\langle e_{2}\right\rangle \oplus\left\langle e_{t+2}, e_{2 t+2}, e_{3 t+2}, \ldots, e_{r t+2}\right\rangle .
\end{aligned}
$$

That is, for any divisor $t \neq 1$ of $n-3$ and for any $\tau \in\{0, \ldots, t-2\}$ we have a $\mathbb{Z}_{t}$-grading of $A$ given by Equation (16) where $A_{\bar{i}}:=A_{i g}$ for any $\bar{i} \in \mathbb{Z}_{t}$ and where $r t=n-3$.

Theorem 19. Let A be a one-parametric filiform Leibniz algebra A of dimension n.

(i) If $n=4$ then any abelian group grading of $A$ is equivalent either to the trivial grading or to the grading (12).

(ii) If $n \geq 5$ then any abelian group grading of $A$ is equivalent either to the trivial grading or to the grading (12) or to one of the gradings in (14) or to one of the gradings in (15) or to one of the gradins in (16).

Proof. We only have to show that, for $n \geq 4$, the grading (12), any grading in (14), any grading in (15) and any grading in (16) are not equivalent. To do that, observe that the grading (12) has one homogeneous component with dimension 1 and one homogeneous component with dimension $n-1$.

Fixed now a divisor $t \geq 2$ of $n-3$ and by denoting $r t=n-3$, we have a grading given by (14) which has one homogeneous component with dimension $r+1$, one homogeneous components with dimension $r+2$ and $t-2$ homogeneous components with dimension $r$; a grading (15) which has one homogeneous component with dimension 1, two homogeneous components with dimension $r+1$ and $t-2$ homogeneous components with dimension $r$; and a grading (16) with either three homogeneous components with dimension $r+1$ and $t-3$ homogeneous components with dimension $r$; or with one homogeneous component with dimension $r+2$, one homogeneous component with dimension $r+1$ and $t-2$ homogeneous components with dimension $r$

From here, the grading (12) is equivalent neither to any grading in (14) nor any grading in (15) and nor any grading in (16) (they have a different number of nonzero homogeneous components). Also, two different gradings in (14), two different gradings in (15) and two different gradings in (16) are not equivalent because they have nonzero homogeneous components with different dimensions. 
Now, let us show that any grading in (14) is not equivalent to any grading in (15). To do that, suppose there exists $t, t^{\prime} \geq 2$ two divisors of $n-3$ such that the $\mathbb{Z}_{t}$-grading of $A$ :

$$
\begin{aligned}
A_{\overline{0}} & =\left\langle e_{t+2}, e_{2 t+2}, e_{3 t+2}, \ldots, e_{r t+2}\right\rangle \oplus\left\langle e_{2}\right\rangle \\
A_{\overline{1}} & =\left\langle e_{1}\right\rangle \oplus\left\langle e_{3}, e_{t+3}, e_{2 t+3}, \ldots, e_{(r-1) t+3}\right\rangle \oplus\left\langle e_{n}\right\rangle \\
A_{\overline{2}} & =\left\langle e_{4}, e_{t+4}, e_{2 t+4}, \ldots, e_{(r-1) t+4}\right\rangle \\
A_{\overline{3}} & =\left\langle e_{5}, e_{t+5}, e_{2 t+5}, \ldots, e_{(r-1) t+5}\right\rangle \\
& \vdots \\
A_{\overline{t-1}} & =\left\langle e_{t+1}, e_{2 t+1}, e_{3 t+1}, \ldots, e_{r t+1}\right\rangle
\end{aligned}
$$

and the $\mathbb{Z}_{t^{\prime}} \times \mathbb{Z}$-grading of $A$

$$
\begin{gathered}
A_{(\overline{1}, 0)}=\left\langle e_{1}\right\rangle \\
A_{(\overline{0}, 1)}=\left\langle e_{3}, e_{t^{\prime}+3}, e_{2 t^{\prime}+3}, \ldots, e_{\left(r^{\prime}-1\right) t^{\prime}+3}\right\rangle \oplus\left\langle e_{n}\right\rangle \\
A_{(\overline{1}, 1)}=\left\langle e_{4}, e_{t^{\prime}+4}, e_{2 t^{\prime}+4}, \ldots, e_{\left(r^{\prime}-1\right) t^{\prime}+4}\right\rangle \\
A_{(\overline{2}, 1)}=\left\langle e_{5}, e_{t^{\prime}+5}, e_{2 t^{\prime}+5}, \ldots, e_{\left(r^{\prime}-1\right) t^{\prime}+5}\right\rangle \\
\vdots \\
A_{\left(\overline{t^{\prime}-2}, 1\right)}=\left\langle e_{t^{\prime}+1}, e_{2 t^{\prime}+1}, e_{3 t^{\prime}+1}, \ldots, e_{r^{\prime} t^{\prime}+1}\right\rangle \\
A_{\left(t^{\prime}-1,1\right)}=\left\langle e_{2}\right\rangle \oplus\left\langle e_{t^{\prime}+2}, e_{2 t^{\prime}+2}, e_{3 t^{\prime}+2}, \ldots, e_{r^{\prime} t^{\prime}+2}\right\rangle
\end{gathered}
$$

are equivalent, where $r t=n-3$ and $r^{\prime} t^{\prime}=n-3$. Since both gradings must have the same number of nonzero homogeneous components, then necessarily

$$
t=t^{\prime}+1 \text {. }
$$

As the grading (18) has an homogeneous component with dimension 1 , then necessarily $r=1$ and so $t=n-3$ in the grading (17). Then we have by Equation (19) that $t^{\prime}=n-4$. Since $t^{\prime}$ divides $n-3$ then necessarily $n-3=2$ and $t^{\prime}=1$, but this contradicts the fact that $t \geq 2$. We conclude that the gradings in (17) and in (18) are not equivalent.

Similar arguments allow us to verify that any grading in (14) is not equivalent to any grading in (16); and that any grading in (15) is not equivalent to any grading in (16).

\section{REFERENCES}

[1] J. Adashev, L.M. Camacho and B.A. Omirov: Central extensions of null-filiform and naturally graded filiform nonLie Leibniz algebras, Journal of Algebra, 479 (2017), 461-486.

[2] Sh. Ayupov and B.A. Omirov: On some classes of nilpotent Leibniz algebras, Siberian Mathematical Journal, 42 (2001), 1, 15-24.

[3] D.W. Barnes: On Levi's theorem for Leibniz algebras, Bulletin of the Australian Mathematical Society, 86 (2012), 2 , $184-185$.

[4] Yu. Bahturin, M. Goze and E. Remm: Group Gradings on Filiform Lie Algebras, Communications in Algebra, 44 (2016), 1, 40-62.

[5] A. J. Calderón Martín, C. Draper and C. Martín-González: Gradings on the Kac superalgebra, Journal of Algebra, 324 (2010), 12, 3249-3261

[6] A. J. Calderón Martín, C. Draper and C. Martín-González and J. M. Sánchez Delgado: Gradings and symmetries on Heisenberg type algebras, Linear Algebra and its Applications, 458 (2014), 463-502. 
[7] A. J. Calderón Martín, C. Martín González and D. Ndoye: On groupoid gradings, Journal of Geometry and Physics, 123 (2018), 61-70.

[8] L. Camacho, I. Kaygorodov, B. Omirov and G. Solijanova: Some solvable cohomologically rigid Leibniz algebras, Journal of Algebra, 560 (2020), 502-520.

[9] J.M. Casas, M. Ladra, B.A. Omirov and I.A. Karimjanov: Classification of solvable Leibniz algebras with nullfiliform nilradical, Linear and Multilinear algebra, 61 (2013), 6, 758-774.

[10] B. Dherin and F. Wagemann: Deformation quantization of Leibniz algebras, Advances in Mathematics, 270 (2015), 21-48.

[11] C. Draper: A non-computational approach to the gradings on $\mathfrak{f}_{4}$, Revista Matemática Iberoamericana, 28 (2012), 1, 273-296.

[12] C. Draper and A. Elduque: Fine gradings on the simple Lie algebras of type E, Note di Matematica, 34 (2014), 1, 53-88.

[13] C. Draper and C. Martín-González: Gradings on $\mathfrak{g}_{2}$, Linear Algebra and Its Applications, 418 (2006), $1,85-111$.

[14] C. Draper and C. Martín-González: Gradings on the Albert algebra and on $\mathfrak{f}_{4}$, Revista Matemática Iberoamericana, 25 (2009), 3, 841-908.

[15] C. Draper, C. Martín-González and A. Viruel: Fine gradings on the Lie algebra $\mathfrak{d}_{4}$, Forum Mathematicum, 22 (2010), 5, 863-877.

[16] C. Draper and A. Viruel: Fine gradings on $\mathfrak{e}_{6}$, Publicacions Matemàtiques, 60 (2016), 1, 113-170.

[17] A. Elduque: Gradings on octonions, Journal of Algebra, 207 (1998), 1, 342-354.

[18] A. Elduque: Jordan gradings on the exceptional simple Lie algebras, Proceedings of the American Mathematical Society, 137 (2009), 12, 4007-4017.

[19] A. Elduque: Fine gradings on simple classical Lie algebras, Journal of Algebra, 324 (2010), 12, 3532-3571.

[20] A. Elduque and M. Kochetov: Gradings on the exceptional Lie algebras $F_{4}$ and $G_{2}$ revisited, Revista Matemática Iberoamericana, 28 (2012), 3, 773-813.

[21] A. Elduque and M. Kochetov: Gradings on simple Lie algebras. Mathematical Surveys and Monographs 189, American Mathematical Society, Providence, RI, 2013.

[22] A. Elduque and M. Kochetov: Gradings on the Lie algebra $D_{4}$ revisited, Journal of Algebra, 441 (2015), $441-474$.

[23] J.R. Gómez, A. Jiménez-Merchán and J. Reyes: Maximum length filiform Lie algebras, Extracta Mathematicae, 16 (2001), 3, 405-421.

[24] J.R. Gómez, A. Jiménez-Merchán and J. Reyes: Quasi-filiform Lie algebras of maximum length, Linear Algebra and its Applications, 335 (2001), 119-135.

[25] N. Ismailov, I. Kaygorodov and Yu. Volkov: The geometric classification of Leibniz algebras, International Journal of Mathematics, 29 (2018), 5, Paper: 1850035.

[26] A. Khudoyberdiyev and B.A. Omirov: Infinitesimal deformations of null-filiform Leibniz superalgebras, Journal of Geometry and Physics, 74 (2013), 370-380.

[27] A. Khudoyberdiyev and B.A. Omirov: Infinitesimal deformations of naturally graded filiform Leibniz algebras, Journal of Geometry and Physics, 86 (2014), 149-163.

[28] B.A. Omirov and I.S. Rakhimov: On Lie-like complex filiform Leibniz algebras, Bulletin of the Australian Mathematical Society, 79 (2009), 391-404

[29] J. Patera and H. Zassenhaus: On Lie gradings. I, Linear Algebra and Its Applications, 112 (1989), 87-159.

[30] P. Zusmanovich: Non-semigroup gradings of associative algebras, Linear Algebra and Its Applications, 523 (2017), $52-58$ 